\title{
Sex differences in use of inhalants by elderly patients with asthma
}

This article was published in the following Dove Press journal:

Clinical Interventions in Aging

10 August 2015

Number of times this article has been viewed

\section{Masahiro Hirose \\ Rieko Kondo \\ Naoaki Ban \\ Kazunobu Kuwabara \\ Mamoru Shiga \\ Takahiko Horiguchi}

Department of Respiratory Medicine II, Fujita Health University School of Medicine, Nagoya, Aichi, Japan
Correspondence: Masahiro Hirose Depatment of Respiraory Medicine II, Fujita Health University School of Medicine, 3-6-10, Otobashi, Nakagawa-ku, Nagoya, 454-85-09, Japan Fax +8I 523235675

Email hiromasa87@yahoo.co.jp
Background: The number of elderly patients with asthma has been increasing in Japan. Treatment for these patients should be provided based on the condition of individual patients. This study was performed to clarify the relationship between inhalation procedure and sex difference in elderly patients with asthma.

Methods: The inhalation procedure was examined in 155 elderly patients with asthma (male: $\mathrm{n}=66$, average age \pm standard deviation: $75.5 \pm 5.65$ years old; female: $n=89$, average age \pm standard deviation: $78.7 \pm 6.87$ years old) during a medical examination.

Results: For the three items that were common to all devices, the percentages of the 155 patients who could/could not perform the actions were examined by separate Fisher's exact tests for males and females. A statistically significant difference $(P=0.007)$ was observed for "breath holding", and more females than males were not able to hold their breath. Although no significant difference was seen in the "accurate number of times of inhalation", females tended to not be able to inhale accurately compared to males $(P=0.072)$.

Conclusion: Our results suggest that elderly female patients with asthma have less understanding of inhaled steroid therapy, compared to elderly male patients. Therefore, it is particularly important to confirm that the correct inhalation procedure is used by elderly female patients with asthma.

Keywords: asthma, elderly, sex, inhalation, therapy

\section{Introduction}

The number of deaths in patients with bronchial asthma in Japan has decreased after introduction of the Guidelines for Prevention and Management of Asthma and with increased use of anti-inflammatory drugs. ${ }^{1}$ The number of deaths due to asthma in Japan in 2011 was 2,060, which is approximately $33 \%$ of the mortality rate 10 years ago. However, the number of elderly patients with asthma has increased with aging of society and treatment of these patients can be difficult due to conditions such as COPD. Bronchial asthma is mainly treated with anti-inflammatory inhaled steroids, but this therapy may be difficult to administer in elderly patients. Therefore, different approaches to treatment of asthma are required for these patients, while confirmation of appropriate inhalation is needed for those who can receive treatment with inhalants. In this context, a recent report suggested the presence of more severe asthma and a lower rate of correct inhalation in female patients. ${ }^{2}$ Here, we report the results of a study in elderly patients with asthma who were outpatients at our hospital, in which the aim was to clarify the reasons for the sex difference in the inhalation procedure.

\section{Methods}

The subjects were 155 adults patients with bronchial asthma who made regular visits to Banbuntane Hotokukai Hospital at Fujita Health University and to related medical 
institutions from September 2010 to March 2011 and gave informed consent for the study. The patients included 66 males (average age \pm standard deviation: $75.5 \pm 5.65$ years old) and 89 females (average age \pm standard deviation: 78.7 \pm 6.87 years old), who did not have dementia, neurological disorders, or muscular disorders. We performed an investigation of the inhalation procedure during a medical examination. The severity of the asthma was mild and persistent or severe and persistent, and the duration of the disease ranged from 1 to 43 years.

The diagnosis of asthma was made by physicians according to JGL2012, ${ }^{1}$ and the instructions on the inhalation of steroids were given by physicians or pharmacists. The patients who had been treated with inhaled corticosteroids for at least 3 months were selected. Four patients with severe persistent asthma were concomitantly taking an oral corticosteroid (prednisolone), and other patients were taking oral corticosteroids as needed at the time of attacks. However, these patients were not being treated with antibiotics.

A test of respiratory function was conducted together with an inspection of the inhalation procedure.

For this study, items on the instructions that were given to maximize the effects of the inhalation therapy and to keep the device clean during routine medical care were inspected. The patients were required to record the "correct number of inhalations", which was an item that was common to all devices, in the asthma diary and whether the patients complied with the required times of inhalation was checked at hospital visits. In patients using more than one device, the use of each device was inspected, and the results were added to the data.

\section{Code of ethics}

The study was approved by the Institutional Review Boards of Fujita Health University (IRB no 14-037). Written informed consent was obtained from all participants.

\section{Results}

The results of the respiratory function tests are shown in Table 1. Statistically significant differences were observed between males and females for vital capacity and forced vital capacity $(P<0.0001)$ and forced expiratory volume in 1 second ( $P=0.007$, Student's $t$-test).

For the three items that were common to all devices, the percentages of the 155 patients who could/could not perform the actions were examined by separate Fisher's exact tests for males and females (Figure 1). A statistically significant difference $(P=0.007)$ was observed for "breath holding", and
Table I Results of the respiratory function tests

\begin{tabular}{lllll}
\hline & $\begin{array}{l}\text { Total } \\
(\mathbf{n}=155)\end{array}$ & $\begin{array}{l}\text { Male } \\
(\mathbf{n}=66)\end{array}$ & $\begin{array}{l}\text { Female } \\
(\mathbf{n}=\mathbf{8 9})\end{array}$ & P-value \\
\hline VC (L) & $2.1 \mathrm{I} \pm 0.79$ & $3.2 \mathrm{I} \pm 0.80$ & $1.79 \pm 0.4 \mathrm{I}$ & $<0.000 \mathrm{I}$ \\
$\mathrm{FVC}(\mathrm{L})$ & $1.97 \pm 0.79$ & $3.00 \pm 0.8 \mathrm{I}$ & $1.67 \pm 0.46$ & $<0.000 \mathrm{I}$ \\
$\mathrm{FEV}_{1}$ (L) & $1.3 \mathrm{I} \pm 0.46$ & $\mathrm{I} .74 \pm 0.68$ & $1.18 \pm 0.28$ & 0.0007 \\
\hline
\end{tabular}

Abbreviations: VC, vital capacity; FVC, forced vital capacity; FEV, forced expiratory volume in I second.

more females than males were not able to hold their breath. Although no significant difference was seen in the "accurate number of times of inhalation", females tended to not be able to inhale accurately compared to males $(P=0.072)$. Next, we examined specific items for individual devices. For 33 patients (male: 14, female: 19) who used a pressurized metered-dose inhaler with spacers, "direction of the inhaler and setting of the spacer" were correctly performed by $93 \%$ of males and $90 \%$ of females, with no significant difference. However, there was a difference of $14 \%$ in "sufficient inhalation", which was observed in $93 \%$ of males and $79 \%$ of females. Cleaning of the spacer was correctly performed by $35 \%$ of males and $58 \%$ of females, showing a much higher rate in female patients (Figure 2).

Among 51 patients (male: 20, female: 31 ) using a Diskus (fluticasone propionate, salmeterol/fluticasone combination) "confirmation of counting of residual amount" was performed by $90 \%$ of males and $87 \%$ of females, while "correct operation of the lever" occurred for $90 \%$ of males and $81 \%$ of females, with no significant difference between the sexes. Sufficient inhalation occurred in $95 \%$ of males and $84 \%$ of females (Figure 3).

For patients using a Turbuhaler (male: 26 , female: 31 ), an "open air duct" was found in $85 \%$ of males and $81 \%$ of females, with no significant difference. However, the rates of three other items ("correct rotation of the inhaler", "vertical setting during rotation", and "sufficient inhalation") were $89 \%, 85 \%$, and $89 \%$ in males and $78 \%, 71 \%$, and $78 \%$ in females, respectively, with each item having approximately a $10 \%$ lower rate in females (Figure 4). The "confirmation of counting of residual amount" was $86 \%$ in males and $80 \%$ in females, which were almost the same percentages.

Among patients using a Twisthaler (male: 16, female: 18) "no vertical setting during rotation" and "insufficient screw of the cap" occurred in $81 \%$ of males and $72 \%$ of females, while "sufficient inhalation" was found in $87 \%$ of males and $72 \%$ of females, indicating a difference of 15\% (Figure 5). The "confirmation of counting of residual amount" was $87 \%$ in males and $82 \%$ in females, which were almost the same percentages. 


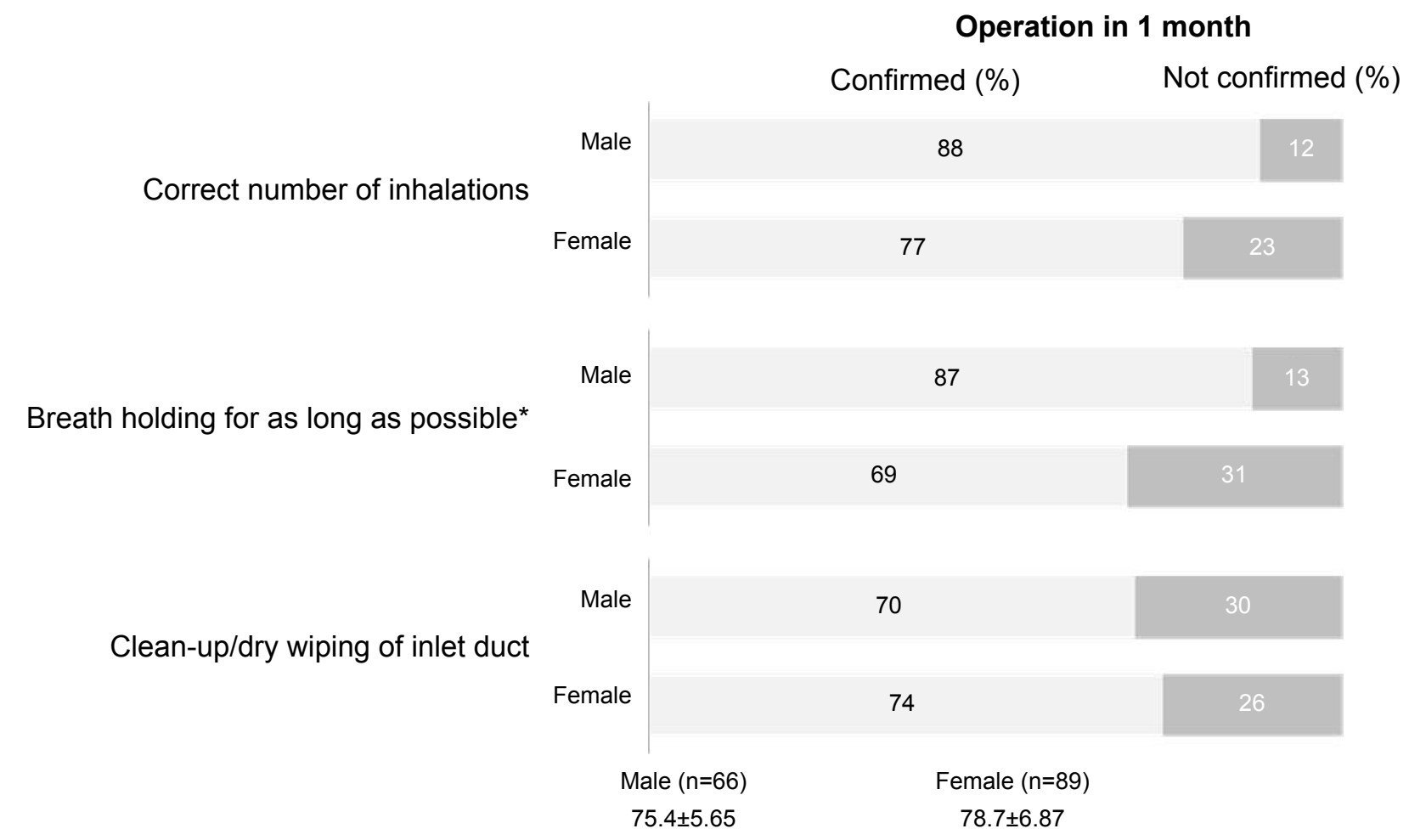

Figure I Results for items common to all inhalation devices.

Notes: $* P=0.007$. Average age \pm standard deviation.

Operation in 1 month

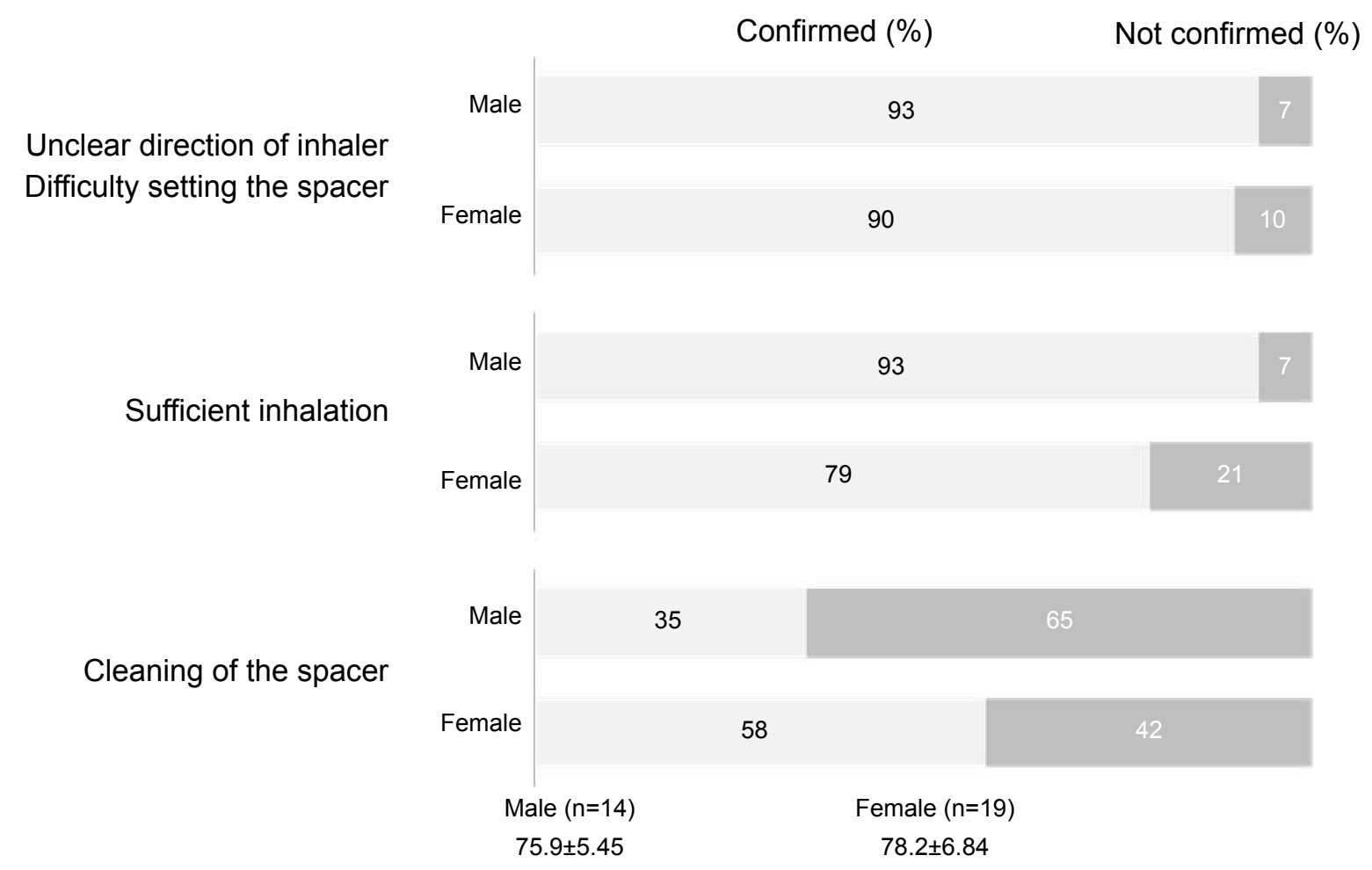

Figure 2 Results for use of a pressurized metered-dose inhaler (pMDI) with spacers.

Note: Average age \pm standard deviation. 


\section{Operation in 1 month}

Confirmation of counting of residual amount

Correct operation of the lever

Second inhalation keeping the cover open No use of the counter

Sufficient inhalation

\section{Confirmation of counting of} residual amount

Air duct kept open

Sufficient inhalation

Figure 3 Results for use of a Diskus.

Note: Average age \pm standard deviation.

\begin{abstract}
Correct rotation of inhaler
Vertical setting during rotation
\end{abstract}

Confirmed (\%)

Female

Male

Female

Male

Female

Male

Female

Male $(n=20)$
90

87

Not confirmed (\%)

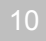

13

81

19

90

10

81

19

\section{5}

84

16

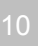

10

10

\section{Operation in 1 month}

$76.1 \pm 6.03$

Confirmed (\%)

Male
Female

86

80

Female $(n=31)$

$78.9 \pm 6.98$ 


\section{Confirmation of counting of residual amount}

\section{No vertical setting during rotation}

\section{Insufficient screw of the cap} (no clicking sound)

\section{Sufficient inhalation}

\section{Discussion}

In Japan, anti-inflammatory treatment with inhaled steroids is widely used in line with the Guidelines for Prevention and Management of Asthma, ${ }^{1}$ and this approach has resulted in an annual decrease in mortality due to asthma. However, most patients who die from asthma are aged 65 years or older, and thus long-term management of asthma in elderly patients has become increasingly important.

According to our previous survey on the preference for drugs for long-term control of asthma, patients who preferred not to use an inhaler were mainly elderly, with $36 \%$ of this group aged $65-74$ years and $43 \%$ aged $75-84$ years. $^{3}$ This seemed to be attributable to the observation that the use of inhalers is not very common among Japanese people and suggests that understanding of inhalation devices and of inhaled steroids may be insufficient, and that it may not be easy for elderly patients to perform the inhalation procedure. However, respiratory function has been found to be significantly improved after providing elderly asthma patients with training in inhalation. ${ }^{4}$ Thus, correct treatment for individual patients can make a major contribution to improvement of asthma control.

In this study, we examined possible sex differences in the inhalation procedure in elderly patients with asthma who

\section{Operation in 1 month}

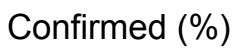

Not confirmed (\%)

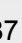

82

28

72

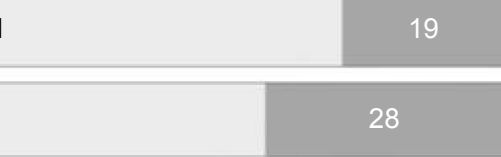

81 19

28

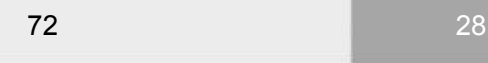

were treated with inhaled steroids. This sex difference in elderly asthma patients could be due to the complication of COPD, and muscle strength of hands and fingers, but these factors were not investigated in the current study.

The results showed that the percentage of males who were able to perform "breath holding" was significantly larger than the percentage of females, and a similar result was observed for the "correct number of inhalations" which is the item that checks the adherence of inhaled corticosteroids (ICS). These findings suggest that men have a deeper understanding of the dosing procedure and the importance of inhaled steroids, while the understanding of them was low in female patients. The possible reasons for this include that Japanese people have a smaller body size than American and European people; the body size of elderly women is even smaller; age-related changes, such as kyphoscoliosis, are common; abdominal breathing is mainly used; and breath holding and inhalation speed are not sufficient in a large percentage of people. No significant differences were observed for the individual devices, the percentage of patients who met the criteria for all items except those related to cleaning the device were higher in males. In particular, the rate of "sufficient inhalation" was approximately $10 \%$ higher in 
males for all devices. This result is similar to the finding of Goodman et $\mathrm{al}^{2}$ that the rate of correct inhalation in females was significantly lower than that in male patients.

Concerning the items related to washing, it was considered likely that females are more familiar with cleaning and washing than males because it is customary in Japan that males work outside their homes, such as at companies, while women work at home. Moreover, a study on the differences in compliance with short-acting beta-agonists (SABA), which is a drug that is used to treat asthma attacks, recently reported a correlation between inhalation compliance and severity. ${ }^{5}$ Our present report was on the use of inhaled steroids that need not to be carried around and that can be used at home for the long-term management of asthma, rather than a drug like SABA, which is used at the time of an attack. Thus, the purpose of the use of inhalants might have been different.

Inhaled steroids are also the first-line drugs for elderly asthma patients, but there may be problems with the inhalation procedure when a physician provides this treatment without clear instructions. In some cases, this may result in reduced effects of the inhalant. The results of this study suggest that provision of clear information on the inhalation procedure is particularly important for elderly female patients. If limited effects of inhaled steroids are likely even after repeated training for inhalation, use of oral antiinflammatory agents such as leukotriene receptor antagonists and transdermal preparations should be considered.

\section{Conclusion}

In conclusion, in this study we examined potential sex differences in the inhalation procedure in elderly asthma patients. Many female patients used an incorrect inhalation procedure, compared to male patients, and thus more training in inhalation is required for female patients. Confirmation of the correct inhalation procedure will be increasingly important for elderly asthma patients with the continuing aging of society.

\section{Disclosure}

The authors report no conflict of interest in this work.

\section{References}

1. Japanese Society of Allergology. Asthma Prevention and Management Guidelines (JGL). Japan: Kyowa Kikaku; 2012.

2. Goodman DE, Israel E, Rosenberg M, Johnston R, Weiss ST, Drazen JM. The influence of age, diagnosis, and gender on proper use of metered-dose inhalers. Am J Respir Crit Care Med. 1994;150(5 Pt 1):1256-1261.

3. Horiguchi T, Kondo R, Ban N, et al. [Survey of type of administration in treatment of asthma in adults in different age groups: problems of asthma in elderly patients]. Prog Med. 2011;31:2469-2471. Japanese.

4. Horiguchi T, Kondo R, Ban N, et al. [Therapeutic approach based on characteristics of asthma in elderly patients: procedure and level of understanding of inhalant drugs]. Allergy Immunity. 2010;17:1086-1093. Japanese.

5. Linder PS, Linder AJ. Gender differences in asthma inhaler. Conn Med. 2014;78(4):207-210
Clinical Interventions in Aging

\section{Publish your work in this journal}

Clinical Interventions in Aging is an international, peer-reviewed journal focusing on evidence-based reports on the value or lack thereof of treatments intended to prevent or delay the onset of maladaptive correlates of aging in human beings. This journal is indexed on PubMed Central, MedLine,

\section{Dovepress}

CAS, Scopus and the Elsevier Bibliographic databases. The manuscript management system is completely online and includes a very quick and fair peer-review system, which is all easy to use. Visit http://www.dovepress. com/testimonials.php to read real quotes from published authors. 